International Journal of Modern Physics B

Vol. 28, No. 15 (2014) 1492002 (1 page)

(C) World Scientific Publishing Company

DOI: $10.1142 /$ S0217979214920027

\title{
Erratum:
}

\section{Casimir torque on two rotating plates}

[Int. J. Mod. Phys. B Vol. 27, No. 14 (2013) 1350066 (19 pages)]

\author{
Xiang Chen \\ Department of Physics, Arizona State University, \\ Tempe, Arizona 85287-1504, USA \\ xchen48@asu.edu
}

Published 24 April 2014

In page 9, there is a term missing in Eq. (36) and should be corrected as shown in the following,

$$
\begin{aligned}
M_{1}\left(V_{d 1 d 2}\right)= & \frac{k_{e} \hbar}{2 \pi} \int_{0}^{\infty} d \omega\left\{\left(\frac{4}{r^{6}}+\frac{9}{r^{10}} x_{1 \perp}^{2} x_{2 \perp}^{2}\right)\right. \\
& \times\left[\left[n_{1}\left(\omega_{+}\right)-n_{2}\left(\tilde{\omega}_{+}\right)\right] \operatorname{Im}\left\{\alpha_{1 \perp}\left(\omega_{+}\right)\right\} \operatorname{Im}\left\{\alpha_{2 \perp}\left(\tilde{\omega}_{+}\right)\right\}\right. \\
& \left.-\left[n_{1}\left(\omega_{-}\right)-n_{2}\left(\tilde{\omega}_{-}\right)\right] \operatorname{Im}\left\{\alpha_{1 \perp}\left(\omega_{-}\right)\right\} \operatorname{Im}\left\{\alpha_{2 \perp}\left(\tilde{\omega}_{-}\right)\right\}\right] \\
& +\frac{9}{r^{10}} x_{1 \perp}^{2} x_{2 \perp}^{2}\left[\left[n_{1}\left(\omega_{+}\right)-n_{2}\left(\tilde{\omega}_{-}\right)\right] \operatorname{Im}\left\{\alpha_{1 \perp}\left(\omega_{+}\right)\right\} \operatorname{Im}\left\{\alpha_{2 \perp}\left(\tilde{\omega}_{-}\right)\right\}\right] \\
& \left.+\frac{36}{r^{10}} x_{1 \perp}^{2} z_{2}^{2}\left[\left[n_{1}\left(\omega_{+}\right)-n_{2}(\omega)\right] \operatorname{Im}\left\{\alpha_{1 \perp}\left(\omega_{+}\right)\right\} \operatorname{Im}\left\{\alpha_{2 \|}(\omega)\right\}\right]\right\} .
\end{aligned}
$$

Such a correction may change one of my original conclusions. With the missing term included, a temperature "induced" torque due to the difference in temperature on two non-rotating plates does not exist.

I acknowledge Arthur Kyasov and George Dedkov by pointing out this mistake. 